3D simulation of interdendritic flow through a $\mathrm{Al}-18 \mathrm{wt} . \% \mathrm{Cu}$ structure captured with X-ray microtomography

This article has been downloaded from IOPscience. Please scroll down to see the full text article.

2012 IOP Conf. Ser.: Mater. Sci. Eng. 27012016

(http://iopscience.iop.org/1757-899X/27/1/012016)

View the table of contents for this issue, or go to the journal homepage for more

Download details:

IP Address: 129.247.247.240

The article was downloaded on 12/01/2012 at 14:53

Please note that terms and conditions apply. 


\title{
3D simulation of interdendritic flow through a Al-18wt.\% Cu structure captured with X-ray microtomography
}

\author{
J Domitner ${ }^{1}$, C Hölzl ${ }^{2}$, A Kharicha ${ }^{1}$, M Wu ${ }^{1}$, A Ludwig ${ }^{1}$, M Köhler ${ }^{3}$ and L Ratke ${ }^{3}$ \\ ${ }^{1}$ Christian Doppler Laboratory for Multiphase Modeling of Metallurgical Processes, \\ University of Leoben, Franz-Josef-Straße 18, 8700 Leoben, Austria \\ ${ }^{2}$ Materials Center Leoben Forschung GmbH, Roseggerstraße 12, 8700 Leoben, Austria \\ ${ }^{3}$ Institute of Materials Physics in Space, German Aerospace Center, Linder Höhe, \\ 51147 Köln, Germany \\ E-mail: josef.domitner@unileoben.ac.at
}

\begin{abstract}
A central parameter to describe the formation of porosity and macrosegregation during casting processes is the permeability of the dendritic mushy zone. To determine this specific feature for a binary Al-18wt.\%Cu alloy, flow simulations based on the Lattice Boltzmann (LB) method were performed. The LB method allows an efficient solving of fluid flow problems dealing with complex shapes within an acceptable period of time. The 3D structure required as input for the simulations was captured with X-ray microtomography, which enables the generation of representative geometries for permeability investigations. Removing the eutectic phase from the measured dataset generated a remaining network of solid primary dendrites. In the simulations, a pressure gradient was applied to force the liquid through the free interdendritic channels. The permeability of the structure was then calculated from the resulting flow velocity pattern using Darcy's law. To examine the influence of different boundary conditions on the results obtained, several simulations were conducted.
\end{abstract}

\section{Introduction}

Melt flow phenomena crucially affect the solidification behaviour of metals. Therefore, controlling the melt flow during casting and solidification processes is essential, since the flow pattern often influences the formation of undesired casting defects (e.g. macrosegregation, cavities, pores). The flow inside a solidifying cast product strongly depends on the permeability of the two-phase region consisting of melt and solid dendrites, the so-called "mushy zone".

For different cooling conditions or melt compositions for example, the morphology and therefore the permeability of this zone may vary distinctly. Hence, several numerical and experimental investigations to characterise the permeability have been conducted in the past [1-8]. If one assumes laminar fluid flow through the dendritic mushy zone, the general relationship between the permeability $K$ and the appearing gradient of pressure $p$ can be described by Darcy's law:

$$
K \nabla p=-\eta \vec{v}_{L}
$$

In equation (1), $\eta$ represents the dynamic viscosity of the liquid passing through the permeable structure. $\vec{v}_{L}$ stands for the uninfluenced superficial velocity of the liquid remote from the structure, the so-called "free-stream velocity", which can be calculated from the velocity pattern developing inside the liquid phase. Therefore, knowing the values of $\vec{v}_{L}, \nabla p$ and $\eta$ enables to estimate the 
permeability $K$ for a certain dendritic structure, just by rearranging equation (1). In the simulations presented, a constant pressure gradient $\nabla p$ was applied to force the liquid of viscosity $\eta$ through a network of primary aluminum dendrites. Finally, it was possible to calculate $K$ based on the occurring velocity pattern.

The numerical simulations and the subsequent permeability calculations were performed for a binary Al-18wt. $\% \mathrm{Cu}$ alloy. This alloy consists of two main phases: the primary Al-dendrites depleted in $\mathrm{Cu}$ and the interdendritic eutectic phase enriched with $\mathrm{Cu}$. These various $\mathrm{Cu}$-contents cause a sufficient density difference, which enables the application of X-ray microtomography [9-10] to capture the interface between both phases. After X-ray measurement, the interdendritic eutectic can be hidden in the acquired dataset. Hence, a network of primary Al-dendrites remains which can then be utilized for further permeability assessment.

\section{Experimental investigations}

\subsection{Sample preparation}

The Al-18wt.\%Cu sample investigated with X-ray microtomography had a size of approximately $1.5 \mathrm{x}$ $1.5 \times 3.0 \mathrm{~mm}$. It was taken from the centre of a calotte-shaped casting, as depicted in figure 1 . The melt to produce this casting had a pouring temperature of $750{ }^{\circ} \mathrm{C}$; special cooling conditions were not determined. The upper end of the sample was tapered. Therefore, the X-rays were able to pass the sample sideward and the radiation was not absorbed by the sample material in this area. That allowed evaluating the influence of background absorption on the measured X-ray intensity data. Further polishing of the sample surfaces was not required.

Then the prepared sample was glued on a thin ceramic stick. The stick length was adapted to position the sample directly in front of the target of the microtomography device, as shown in figure 2. This arrangement ensured the $\mathrm{Al}-18 \mathrm{wt} . \% \mathrm{Cu}$ sample to remain inside the $\mathrm{X}$-ray cone when the sample holder rotated during the measurement. Using a ceramic instead of a metal stick was favourable, since the thermal expansion coefficient of ceramics is usually lower. That reduces the error due to thermal expansion, if the stick warms up during the microtomography measurement.

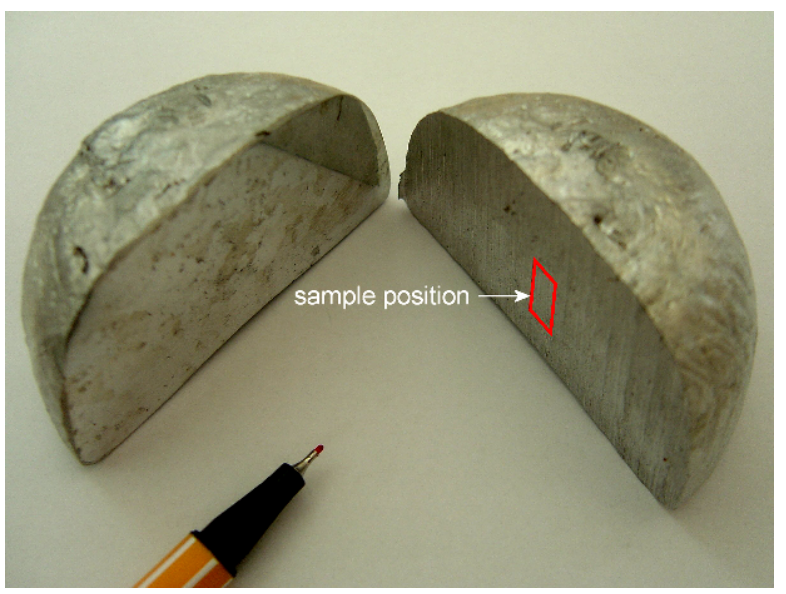

Figure 1. Position of the investigated sample inside the calotte-shaped casting

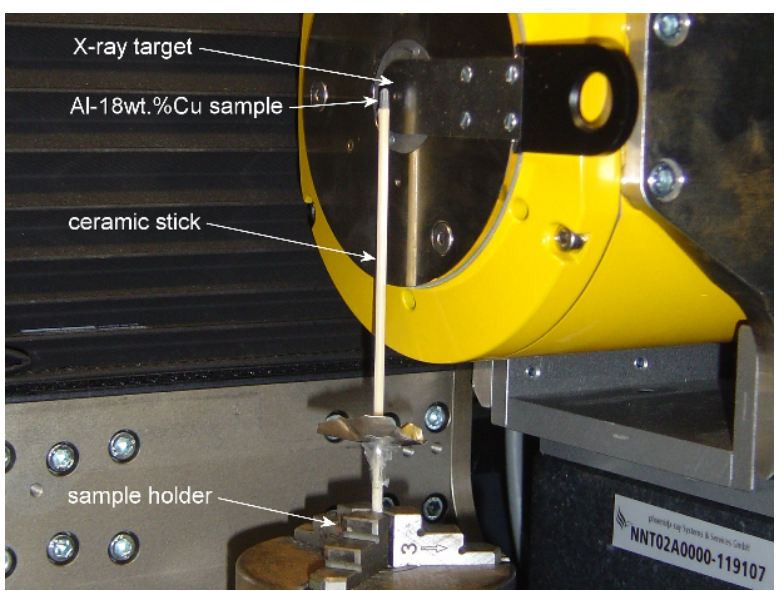

Figure 2. Sample positioned for measurement inside the X-ray microtomography device

\subsection{Data acquisition and $3 D$ reconstruction}

The measurements were performed with a phoenixlx-ray nanotom computer tomograph (CT). The basic CT operation parameters to investigate the Al-18wt.\% Cu sample material are summarised in table 1. After capturing all two-dimensional tomography images, the subsequent reconstruction of the three-dimensional dendritic structure was completed directly at the CT device. 
Table 1. Basic settings for the X-ray microtomography measurements

\begin{tabular}{lc}
\hline X-ray tube voltage & $130 \mathrm{kV}$ \\
X-ray tube current & $100 \mu \mathrm{A}$ \\
Distance between target and detector & $500 \mathrm{~mm}$ \\
Distance between target and sample holder rotation axis & $12 \mathrm{~mm}$ \\
Achieved resolution & $1.2 \mu \mathrm{m}$ \\
Sample holder rotation steps (= number of reconstruction images) & 1000 \\
Number of averaging images acquired per rotation step & 4 \\
Number of skip images acquired per rotation step & 1 \\
Integration time per image & $5 \mathrm{~s}$ \\
\hline
\end{tabular}

\subsection{Data preparation for simulation}

The commercial software package VGStudio Max 1.2.1 was used to visualise the obtained sample structure and to prepare its volume data for the succeeding simulations. First, the number of discrete volume elements ("voxels") was reduced by merging adjacent elements. Thus, the edge length of each voxel doubled from $1.2 \mu \mathrm{m}$ to $2.4 \mu \mathrm{m}$. Each of these small cubic voxels had a certain X-ray intensity value. The higher the local density of the investigated material, the lower was the intensity value of the corresponding voxel. Hence, the density difference between the primary dendrites (low density) and the interdendritic eutectic (higher density) resulted in two different ranges of X-ray intensities. Hiding all voxels within a certain range enabled visualising only one of both phases.

For instance in figure 3, only the primary dendritic phase is shown. The depicted cube consists of $375 \times 375 \times 375$ voxels. Thus, with an edge size of $2.4 \mu \mathrm{m} / \mathrm{voxel}$, the cube has a total edge length of $900 \mu \mathrm{m}$ and a dendritic volume fraction of approx. $64 \%$. Notice the large dendrite trunk, which is coincidentally cut by the boundary plane along its main axis. To reduce the surface roughness of the obtained structure, the intensity values had been smoothed with a three-dimensional Gaussian filter comprising of $7 \times 7 \times 7$ voxels. Finally, all values were stored inside a *txt-file which is suitable for being processed in the used simulation software. For that purpose it was necessary to classify the data. All of the intensity values representing the eutectic phase were assigned to be 1 , while all of the remaining values representing the dendritic phase were assigned to be 0 .

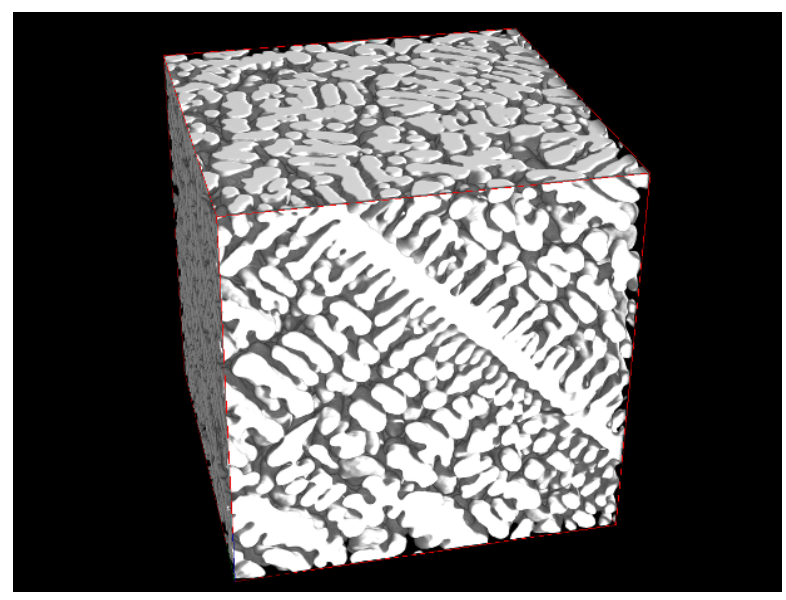

Figure 3. CT reconstruction of the dendritic phase

\section{Fluid flow simulations}

To investigate the interdendritic fluid flow passing through the dendritic structure, the open source library Palabos was utilized [11], which provides a modular $C++$ written code based on the Lattice Boltzmann (LB) method [12-16]. This method is advantageous for solving fluid flow problems in complex shaped structures containing irregular boundaries within reasonable calculation time. 


\subsection{Numerical model}

Lattice Boltzmann (LB) methods can be regarded as discretised modifications of the Boltzmann equation. According to the discretization of Bhatnagar-Gross-Krook the LB equation can be written as

$$
f_{i}(\vec{x}+\Delta \vec{x}, t+\Delta t)=f_{i}(\vec{x}, t)-\frac{\Delta t}{\tau}\left(f_{i}(\vec{x}, t)-f_{i}^{e q}(\vec{x}, t)\right) .
$$

Equation (2) describes the movement of particle populations at discrete time instances along the directions $i$ inside a lattice. The number of possible propagation directions $i$ depends on the chosen LB model. For the simulations presented here, a three-dimensional model (D3) considering 19 directions (Q19) was applied. Therefore, $i=0,1,2, \ldots 18$ in this D3Q19 model. While $f_{i}$ represents the actual distribution function of the particle populations at the spatial position $\vec{x}$ and at time $t, f_{i}^{e q}$ denotes the equilibrium distribution function of these populations. $\tau$ stands for the dimensionless relaxation time of the fluid and $\Delta \vec{x}$ characterises the lattice spacing. If we define the particle velocity vector $\vec{c}_{i}=\Delta \vec{x} / \Delta t$ and if we set the discrete time step $\Delta t$ to unity, equation (2) simplifies to

$$
\begin{aligned}
& f_{i}\left(\vec{x}+\vec{c}_{i}, t+1\right)=f_{i}(\vec{x}, t)-\frac{1}{\tau}\left(f_{i}(\vec{x}, t)-f_{i}^{e q}(\vec{x}, t)\right), \\
& \text { with } f_{i}^{e q}=w_{i} \rho\left(1+3 \vec{c}_{i} \cdot \vec{u}+\frac{9}{2}\left(\vec{c}_{i} \cdot \vec{u}\right)^{2}-\frac{3}{2} \vec{u} \cdot \vec{u}\right) .
\end{aligned}
$$

The weighting factors $w_{i}$ in equation (4) depend on the chosen LB model and on the directions $i$. For the D3Q19 model, $w_{0}=1 / 3, w_{1-6}=1 / 18$ and $w_{7-18}=1 / 36$ [15]. $\rho$ is the macroscopic fluid density and $\vec{u}$ is the fluid velocity. For each lattice cell, $\rho$ and $\vec{u}$ can be calculated with equations (5) and (6):

$$
\rho=\sum_{i=0}^{Q-1} f_{i}, \quad \rho \vec{u}=\sum_{i=0}^{Q-1} f_{i} \vec{c}_{i}
$$

\subsection{Simulation grid}

For the performed simulations, a domain comprising $200 \times 200 \times 200$ voxels was taken from the structure shown previously in figure 3 . Therefore, the cubic simulation domain had an edge length of $480 \mu \mathrm{m}$. To each of the 8 million voxels one element of the regular LB volume grid was assigned, which had the value of 0 (dendritic phase) or 1 (interdendritic eutectic phase), respectively. Hence, the created LB grid consisted of two different parts, one part for each phase. In the present simulations the flow through the eutectic part of the grid was investigated, which was assumed to remain liquid just before reaching the eutectic temperature.

\subsection{Boundary conditions}

In the current simulations, the influence of different boundary conditions on the simulation results was investigated. For this purpose two simulation cases, A and B, considering an identical dendritic structure but different boundary conditions were compared. These conditions are listed in table 2 .

Table 2. Boundary conditions for the simulation cases A and B

\begin{tabular}{ccc}
\hline Boundary No. & Simulation case A & Simulation case B \\
\hline $\mathrm{x} 1, \mathrm{x} 2$ & periodic & periodic \\
$\mathrm{y} 1, \mathrm{y} 2, \mathrm{z} 1, \mathrm{z} 2$ & periodic & non-slip wall \\
\hline
\end{tabular}

The periodic boundary condition enables the flow which leaves the simulation domain through a certain boundary face " 1 " to enter the domain again at the opposite boundary face " 2 ". Hence, the developing flow pattern is uninfluenced by non-linear effects, which may occur if conventional inlet boundary conditions are used. However, applying the periodic condition requires pairs of boundary faces having solid and liquid lattice cells at the same position. To ensure this, the simulation domain was mirrored in each of the coordinate directions $(x, y, z)$ for case A or only along the main flow 
direction $x$ for case B, respectively. Thus, case A represents an indefinitely extended structure, while case B represents a channel of square cross-section filled with dendrites similar to configurations of experimental permeability investigations [8]. Due to mirroring, the simulation domain size increased from 8 million to 64 million grid elements for case A and to 16 million grid elements for case B.

Additionally, two different pressure gradients of $10^{-3} \mathrm{~Pa} / \mathrm{mm}$ and of $10^{-1} \mathrm{~Pa} / \mathrm{mm}$ were applied along the main flow direction $x$ for each simulation case. These low pressure gradients were chosen to achieve laminar flow (Reynolds number $\mathrm{Re}<1$ ) through the structure. To consider the gradients in the LB simulations, constant body forces acting on each of the liquid lattice elements were introduced. For the interface between solid and liquid the halfway bounce back boundary condition was used [16].

\section{Results and discussion}

Due to the applied pressure gradient the liquid was forced to flow through the interdendritic channels. Hence, depending on the applied pressure gradients and on the boundary conditions, slightly different velocity patterns inside the liquid developed. One of the velocity patterns obtained for simulation case $\mathrm{A}$ at the mirror plane in $x$-direction is exemplarily depicted in figure 4 . Figure 5 shows the velocity difference between simulation cases A and B at the identical position. The coloured areas in figures 4 and 5 indicate the liquid velocity, while the grey areas show the dendritic network at the mirror plane in $x$-direction of the domain. Notice that the colour scales of both figures differ by a factor of 10 .

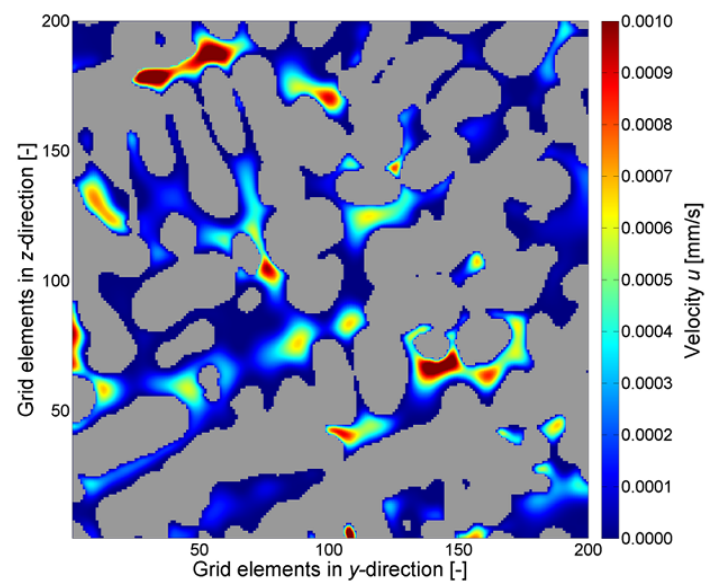

Figure 4. Velocity pattern for simulation case A at the mirror plane in $x$-direction of the simulation domain (applied pressure gradient: $10^{-1} \mathrm{~Pa} / \mathrm{mm}$ )

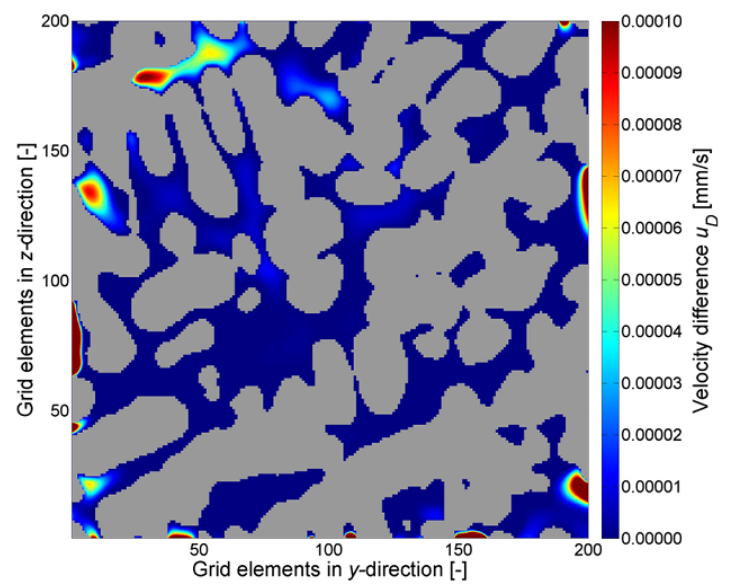

Figure 5. Velocity difference between simulation cases $\mathrm{A}$ and $\mathrm{B}$ at the mirror plane in $x$-direction (applied pressure gradient: $10^{-1} \mathrm{~Pa} / \mathrm{mm}$ )

Determining the permeability according to equation (1) requires knowing the free-stream velocity $\vec{v}_{L}$. As given in equation (7), it is possible to calculate $\vec{v}_{L}$ from all liquid cell velocities at the mirror plane in $x$-direction. $N_{L}$ and $N_{S}$ denote the total numbers of liquid and solid lattice cells at the mirror plane, while $\vec{u}_{j}$ represents the local liquid velocity obtained inside a certain lattice cell $j$.

$$
\vec{v}_{L}=\frac{1}{N_{L}+N_{S}} \cdot \sum_{j=1}^{N_{L}} \vec{u}_{j}
$$

The calculated permeability values are summarised in table 3 for both simulation cases, A and B. It is obvious that the permeability $K$ of the dendritic structure does not depend on the applied pressure gradient. Laminar flow provided, $K$ remains constant for each simulation case, since the free-stream velocity increases proportional to the pressure. Comparing the results of simulation cases $\mathrm{A}$ and $\mathrm{B}$ shows a permeability difference of approx. $5 \%$. Notice that instead of being a scalar, $K$ should generally be a direction-dependent rank-two tensor for a particular three-dimensional structure. In the current simulations the eigenvectors of this tensor were not expected to be aligned with the direction of the applied pressure gradient. However, since the structure includes a large number of flow 
channels, it is not surprising that the resulting mean liquid velocity is quasi parallel to the applied pressure gradient. As represented previously in figure 5, velocity differences between both cases occur mainly at the boundaries, while they are negligible inside of the simulation domain.

The permeability values obtained in the current study and summarized in table 3 show a good agreement with results presented in [1-7], while they differ by an order of $10^{-4}$ from the results given in [8]. Nevertheless, discussing this discrepancy is beyond the scope of this paper.

Table 3. Free stream velocity and permeability calculated from the LB simulation results

\begin{tabular}{lcccc}
\hline & \multicolumn{2}{c}{ Simulation case A } & \multicolumn{2}{c}{ Simulation case B } \\
\hline Applied pressure gradient & $10^{-3} \mathrm{~Pa} / \mathrm{mm}$ & $10^{-1} \mathrm{~Pa} / \mathrm{mm}$ & $10^{-3} \mathrm{~Pa} / \mathrm{mm}$ & $10^{-1} \mathrm{~Pa} / \mathrm{mm}$ \\
Calculated free-stream velocity & $5.898 \cdot 10^{-7} \mathrm{~mm} / \mathrm{s}$ & $5.898 \cdot 10^{-5} \mathrm{~mm} / \mathrm{s}$ & $5.622 \cdot 10^{-7} \mathrm{~mm} / \mathrm{s}$ & $5.622 \cdot 10^{-5} \mathrm{~mm} / \mathrm{s}$ \\
Calculated permeability & $2.949 \cdot 10^{-6} \mathrm{~mm}^{2}$ & $2.949 \cdot 10^{-6} \mathrm{~mm}^{2}$ & $2.811 \cdot 10^{-6} \mathrm{~mm}^{2}$ & $2.811 \cdot 10^{-6} \mathrm{~mm}^{2}$ \\
\hline
\end{tabular}

\section{Conclusions}

In the present work, the Lattice Boltzmann simulation method is used to determine the permeability of a dendritic $\mathrm{Al}-18 \mathrm{wt} . \% \mathrm{Cu}$ structure, which has been captured previously with computed tomography. The influence of different boundary conditions on the obtained interdendritic velocity pattern and therefore on the permeability of the dendritic structure is investigated in two simulation cases. Both of these cases consider laminar fluid flow. Although the performed simulations indicate that non-slip wall boundaries may affect the obtained permeability results, this influence can be neglected for the considered simulation domain size. Hence, using wall boundaries instead of periodic boundaries is advisable for the current structure consisting sufficient free flow channels. Then the structure must be mirrored only once to apply the periodic condition in main flow direction. That restricts the number of lattice cells and reduces the required calculation time. Furthermore, the wall boundary configuration is similar to setups used for experimental permeability investigations. However, to examine the influence of non-slip wall boundaries on flow patterns inside of smaller simulation domains and for different solid fractions is an ongoing work.

\section{Acknowledgment}

The authors would like to thank Mr. Georg Nunner and Mr. Gerhard Hochleithner from the University of Leoben for alloying and casting the sample material investigated.

\section{References}

[1] Poirier D R 1987 Metall. Trans. B 18B 245-55

[2] Bhat M S, Poirier D R and Heinrich J C 1995 Metall. Mater. Trans. B 26B 1049-56

[3] Heinrich J C and Poirier D R 2004 C. R. Mecanique 332 429-45

[4] Brown S G R, Spittle J A, Jarvis D J and Walden-Bevan R 2002 Acta Mater. 50 1559-69

[5] Bernard D, Nielsen Ø, Salvo L and Cloetens P 2005 Mater. Sci. Eng. A 392 112-20

[6] Fuloria D, Lee P D and Bernard D 2008 Mater. Sci. Eng. A 494 3-9

[7] Khajeh E and Maijer D M 2010 Mater. Sci.Tech.Ser. 26 1469-76

[8] Khajeh E and Maijer D M 2010 Acta Mater. 58 6334-44

[9] Maire E, Buffière J Y, Salvo L, Blandin J J, Ludwig W, Létang J M 2001 Adv. Eng. Mater.3 539-46

[10] Salvo L, Cloetens P, Maire E, Zabler S, Blandin J J, Buffière J Y, Ludwig W, Boller E, Bellet D and Josserond C 2003 Nucl. Instrum. Meth. B 200 273-86

[11] http://www.lbmethod.org/palabos/documentation.userguide/index.html

[12] Succi S 2001 The Lattice Boltzmann Equation for Fluid Dynamics and Beyond (Oxford: Clarendon Press)

[13] Chen S and Doolen G D 1998 Annu. Rev. Fluid Mech. 30 329-64

[14] Feng Y T, Han K and Owen D R J 2007 Int. J. Numer. Meth. Engng. 72 1111-34

[15] Qian Y H, D'Humieres D and Lallemand P 1992 Europhys. Lett. 17 479-84

[16] Ziegler D P 1993 J. Stat. Phys. 71 1171-77 